\title{
The patterns of traveling wave on shallow water modeled by Green-Naghdi equation
}

\author{
Haitong $\mathrm{Wei}^{*}$ \\ Department of Mathematics, Northeast Petroleum University, Daqing 163318, China
}

\begin{abstract}
The Green-Naghdi equations are a shallow water waves model which play important roles in nonlinear wave fields. By using the trial equation method and the Complete discrimination system for the polynomial we obtained the classification of travelling wave patterns. Among those patterns, new singular patterns and double periodic patterns are obtained in the first time. And we draw the graphs which help us to understand the dynamics behaviors of the Green-Naghdi model intuitionally.
\end{abstract}

Keywords: Green-Naghdi equations, Soliton, Shallow water waves, Trial equation method.

\section{Introduction}

Green-Naghdi equations is a shallow water waves model which can be written as following ${ }^{[1]}$

$$
\begin{gathered}
v_{t}+v v_{x}+u_{x}=\frac{1}{3 u}\left[u^{3}\left(v v_{x x}+v_{x t}-v_{x}^{2}\right)\right]_{x}, \\
u_{t}+(u v)_{x}=0,
\end{gathered}
$$

where $\mathrm{u}(\mathrm{x}, \mathrm{t})$ is the free upper surface and $\mathrm{v}(\mathrm{x}, \mathrm{t})$ is the horizontal velocity of the fluid. Green-Naghdi model describes a kind weekly dispersive nonlinear shallow 1water waves motion which can simulate the propagation of solitons in dispersive media. Compared weekly dispersive nonlinear equations named Boussinesq equations basing small amplitude assumption, Green-Naghdi equations apply to big amplitude question, thus Green-Naghdi equations have more extensive application. In 1953, Serre ${ }^{[2]}$ deduced one-dimensional weekly dispersive shallow water waves model in even bottom. In 1969, Su and Gardner ${ }^{[3]}$ gained same shallow water waves equations by different method. In 1976, Green and Naghdi $^{[4]}$ deduced two-dimensional weekly dispersive shallow water waves equations system (1) and (2) namely Green-Naghdi equations now. In 1987, Santos $^{[5]}$ et al obtained one-dimensional Green-Naghdi model in uneven bottom. In 2010, Dias and Milewski ${ }^{[6]}$ generalized the equations to general form. Due to the im portance of the equations for shallow water waves in physics, the Green-Naghdi equations have been studied by various

\footnotetext{
*Corresponding author: haitongwei217@,126.com
} 
methods. For example, in 2010, Metayer and Gavrilyuk ${ }^{[7]}$ referred a mix-numerical method to deal with the movement of solitons. In 2011, Bonneton et al referred two methods to solve the equations including finite volume method and mix method of finite volume and finite differences. In this paper, we adopt the trial equation method ${ }^{[8-16]}$ to solve the model, and obtain a series of solutions including solitary waves pattern, blow up patterns, periodic wave patterns and periodic blow-up patterns and double periodic wave patterns. ${ }^{[1]}$ Among those, we confirm two new patterns which are singular patterns and double periodic patterns. In addition, we draw the graphs of some patterns to show the dynamical behaviors of the patterns intuitively. The paper is organized as follows:

In section two, we introduce reduction of Green-Naghdi equations. In section three, we give different patterns of travelling wave. In section four, we obtain the expression of the solutions and draw the graphs of some solutions. In last section, we offer the simply conclusion.

\section{Reduction of Green-Naghdi equations}

We take the traveling wave transformation $\mathrm{u}=\mathrm{u}(\xi), \mathrm{v}=\mathrm{v}(\xi), \xi=\mathrm{x}+\omega \mathrm{t}$ and set them into equation(1) and (2) to get

$$
\begin{gathered}
3 u\left[\omega v^{\prime}+v v^{\prime}+u^{\prime}=\left[u^{3}\left(v v^{\prime \prime}+\omega v^{\prime \prime}\right)-\left(v^{\prime}\right)^{2}\right]_{x},\right. \\
u=\frac{C_{0}}{\omega+v},
\end{gathered}
$$

where $\mathrm{C}_{0}$ is a constant .Then,setting (4) into (3) and integrating once, we gain

$$
C_{0}^{3}\left(v^{\prime}\right)^{2}-C_{0}^{3}(\omega+v) v^{\prime \prime}+3 C_{0} v(\omega+v)^{3}+\frac{3}{2} C_{0}^{2}(\omega+v)-C_{1}(\omega+v)^{3}=0,
$$

where $\mathrm{C}_{1}$ is an integral constant. Next, we take the trial equation to solve the equation (5). It is just like

$$
v^{\prime \prime}=a_{m} v^{m}+a_{m-1} v^{m-1}+\ldots+a_{2} v^{2}+a_{1} v+a_{0}
$$

We can get $\mathrm{m}=3$ with the balance principle obviously. Then we can obtain

$$
\left(v^{\prime}\right)^{2}=\frac{a_{3}}{2} v^{4}+\frac{2 a_{2}}{3} v^{3}+a_{1} v^{2}+2 a_{0} v+d_{0} \text {. }
$$

where $d_{0}$ is an integral constant. Then setting (7) into(5),

we get

$$
r_{4} v^{4}+r_{3} v^{3}+r_{2} v^{2}+r_{1} v+r_{0}=0
$$

where 


$$
\left\{\begin{array}{l}
r_{4}=3 C_{0}-\frac{a_{3} C_{0}^{3}}{2} \\
r_{3}=9 C_{0} \omega-a_{3} C_{0}^{3} \omega-\frac{a_{2} C_{0}^{3}}{3}-C_{1} \\
r_{2}=9 C_{0} \omega^{2}-a_{2} C_{0}^{3} \omega-3 C_{1} \omega \\
r_{1}=a_{0} C_{0}^{3}-a_{1} C_{0}^{3} \omega+3 C_{0} \omega^{3}+\frac{3}{2} C_{0}^{2}-3 C_{1} \omega^{2} \\
r_{0}=C_{0}^{3} d_{0}-a_{0} C_{0}^{3} \omega+\frac{3}{2} C_{0}^{2} \omega-C_{1} \omega^{3}
\end{array} .\right.
$$

Then we let $\mathrm{ri}=0(\mathrm{i}=0, \ldots, 4)$, and give

$$
\left\{\begin{array}{l}
a_{0}=\frac{d}{\omega}+\frac{3}{2 C_{0}}-\frac{C_{1} \omega^{2}}{C_{0}^{3}} \\
a_{1}=\frac{d}{\omega^{2}}+\frac{3}{C_{0} \omega}+\frac{3 \omega^{2}}{C_{0}^{2}}-\frac{4 C_{1} \omega}{C_{0}^{3}} \\
a_{2}=\frac{9 \omega}{C_{0}^{2}}-\frac{3 C_{1}}{C_{0}^{3}} \\
a_{3}=\frac{6}{C_{0}^{2}}
\end{array}\right.
$$

Setting (10) into (7) gives

$$
\left(v^{\prime}\right)^{2}=\frac{3}{C_{0}^{2}} v^{4}+\left(\frac{6 \omega}{C_{0}^{2}}-\frac{2 C_{1}}{C_{0}^{3}}\right) v^{3}+\left(\frac{d_{0}}{\omega^{2}}+\frac{3}{C_{0} \omega}+\frac{3 \omega^{2}}{C_{0}^{2}}-\frac{4 C_{1} \omega}{C_{0}^{3}}\right) v^{2}+\left(\frac{2 d_{0}}{\omega}+\frac{3}{C_{0}}-\frac{2 C_{1} \omega^{2}}{C_{0}^{3}}\right) v+d_{0} .
$$

Furthermore, we take the following transformation

$$
\left\{\begin{array}{l}
\mathrm{v}=T-\left(\frac{C_{1}}{6 C_{0}}-\frac{\omega}{2}\right) . \\
\xi_{1}=\frac{\sqrt{3}}{C_{0}} \xi
\end{array} .\right.
$$

Setting (12) into (11), we gain the equation

$$
\left(T^{\prime}\right)^{2}=F(T)=T^{4}+p T^{2}+q T+r
$$

where

$$
\begin{gathered}
p=-\frac{\omega}{2}+\frac{C_{0}}{\omega}+\frac{C_{0}^{2} d_{0}}{3 \omega^{2}}-\frac{C_{1} \omega}{3 C_{0}}-\frac{C_{1}^{2}}{6 C_{0}^{2}}, \\
q=-\omega^{3}-2 C_{0}-\frac{C_{1}^{3}}{27 C_{0}^{3}}+\frac{5 C_{1} \omega^{2}}{3 C_{0}}+\frac{C_{1}^{2} \omega}{3 C_{0}}+\frac{2 C_{1} C_{0} d_{0}}{9 \omega^{2}}+\frac{2 C_{1}}{3 \omega}-\frac{8 C_{1}^{2} \omega}{9 C_{0}^{2}} \\
r=-\frac{\omega^{4}}{2}+d_{0}+\frac{5 C_{1} \omega^{3}}{4 C_{0}}-\frac{23 C_{1} \omega^{2}}{72 C_{0}^{2}}-\frac{\omega C_{1}^{3}}{108 C_{0}^{3}}-\frac{C_{1}^{4}}{432 C_{0}^{4}}-\frac{13 C_{0}^{2} d_{0}}{12}-\frac{7 C_{0} \omega}{4}+\frac{7 C_{1} C_{0} d_{0}}{18 \omega}+\frac{C_{1}}{2}+\frac{C_{1}^{2} d_{0}}{108 \omega^{2}}+\frac{C_{1}^{2}}{36 C_{0} \omega} .
\end{gathered}
$$

Thus, we can write (11) as an integral form

$$
\int \frac{d T}{\sqrt{T^{4}+p T^{2}+q T+r}}= \pm\left(\xi_{1}-\xi_{0}\right) .
$$


Its the complete discrimination system for polynomial $\mathrm{F}(\mathrm{T})$ is given as ${ }^{[17-20]}$

$$
\left\{\begin{array}{l}
E_{2}=9 p^{2}-32 p q \\
D_{1}=4 \\
D_{2}=-p \\
D_{3}=-2 p^{3}+8 p r-9 q^{2} \\
D_{4}=-p^{3} q^{2}+4 p^{4} r+36 p q^{2} r-32 p^{2} r^{2}-\frac{27}{4} q^{4}+64 r^{3}
\end{array} .\right.
$$

In next section, we classify all solutions of equation (16).

\section{Patterns of travelling wave}

Becase of $u=\frac{C_{0}}{\omega+v}$, we only give the solutions of $u$.

Solutions I: When $D_{2}>0, D_{3}>0, D_{4}=0, F(v)=(v-\lambda)^{2}(v-\tau)(v-\kappa)$, we get

$$
\begin{gathered}
u_{1}=\frac{C_{0}}{\omega+\frac{\kappa-\frac{\lambda-\tau}{\lambda-\kappa} \operatorname{coth}^{2} \frac{2}{ \pm\left(\xi_{1}-\xi_{0}\right) \sqrt{(\lambda-\tau)(\lambda-\kappa)}} \tau}{1-\frac{\lambda-\tau}{\lambda-\kappa} \operatorname{coth}^{2} \frac{2}{ \pm\left(\xi_{1}-\xi_{0}\right) \sqrt{(\lambda-\tau)(\lambda-\kappa)}}}}, \\
u_{2}=\frac{C_{0}}{\omega+\frac{\kappa-\frac{\lambda-\tau}{\lambda-\kappa} \tanh ^{2} \frac{ \pm\left(\xi_{1}-\xi_{0}\right) \sqrt{(\lambda-\tau)(\lambda-\kappa)}}{2} \tau}{1-\frac{\lambda-\tau}{\lambda-\kappa} \tanh ^{2} \frac{ \pm\left(\xi_{1}-\xi_{0}\right) \sqrt{(\lambda-\tau)(\lambda-\kappa)}}{2}}} \\
u_{3}=\frac{C_{0}}{\omega+\frac{\tau-\lambda}{\frac{\lambda-\kappa}{\tau-\lambda} \tan ^{2}\left[\left(\xi_{1}-\xi_{0}\right) \frac{\sqrt{(\lambda-\kappa)(\tau-\lambda)}}{2}\right] \tau-\kappa}}
\end{gathered}
$$

Solution II: When $D_{2}>0, D_{3}=0, D_{4}=0, E_{2}=0, F(v)=(v-\mu)^{3}(v-\rho)$, we obtain

$$
u_{4}=\frac{C_{0}}{\omega+\mu+\frac{4(\mu-\rho)}{(\rho-\mu)^{2}\left(\xi_{1}-\xi_{0}\right)^{2}-4}} .
$$

Solutions

III:

When $D_{4}>0, D_{3}>0, D_{1}>0, F(v)=\left(v-\beta_{1}\right)\left(v-\beta_{2}\right)\left(v-\beta_{3}\right)\left(v-\beta_{4}\right)$, we get 


$$
\begin{gathered}
u_{5}=\frac{C_{0}}{\omega+\frac{\beta_{2}\left(\beta_{1}-\beta_{4}\right) \operatorname{sn}^{2}\left(\frac{\sqrt{\left(\beta_{1}-\beta_{3}\right)\left(\beta_{2}-\beta_{4}\right)}}{2}\left(\xi_{1}-\xi_{0}\right), m\right)-\beta_{1}\left(\beta_{2}-\beta_{4}\right)}{\left(\beta_{1}-\beta_{4}\right) s n^{2}\left(\frac{\sqrt{\left(\beta_{1}-\beta_{3}\right)\left(\beta_{2}-\beta_{4}\right)}}{2}\left(\xi_{1}-\xi_{0}\right), m\right)-\left(\beta_{2}-\beta_{4}\right)}} \\
u_{6}=\frac{C_{0}}{\omega+\frac{\beta_{4}\left(\beta_{2}-\beta_{3}\right) \operatorname{sn}^{2}\left(\frac{\sqrt{\left(\beta_{1}-\beta_{3}\right)\left(\beta_{2}-\beta_{4}\right)}}{2}\left(\xi_{1}-\xi_{0}\right), m\right)-\beta_{3}\left(\beta_{2}-\beta_{4}\right)}{\left(\beta_{2}-\beta_{3}\right) \operatorname{sn}^{2}\left(\frac{\sqrt{\left(\beta_{1}-\beta_{3}\right)\left(\beta_{2}-\beta_{4}\right)}}{2}\left(\xi_{1}-\xi_{0}\right), m\right)-\left(\beta_{2}-\beta_{4}\right)}}
\end{gathered}
$$

where

$$
m^{2}=\frac{\left(\beta_{1}-\beta_{4}\right)\left(\beta_{2}-\beta_{3}\right)}{\left(\beta_{1}-\beta_{3}\right)\left(\beta_{2}-\beta_{4}\right)} .
$$

Solution IV: When $D_{4}>0, D_{2} D_{3} \leq 0, F(v)=\left[\left(v-h_{1}\right)^{2}+s_{1}{ }^{2}\right]\left[\left(v-h_{2}\right)^{2}+s_{2}{ }^{2}\right]$

$$
u_{7}=\frac{C_{0}}{\omega+\frac{\left.a_{1} \operatorname{sn}\left(\chi\left(\xi_{1}-\xi_{0}\right), v\right)+a_{2} \operatorname{cn}\left(\chi\left(\xi_{1}-\xi_{0}\right), v\right)\right)}{a_{3} \operatorname{sn}\left(\chi\left(\xi_{1}-\xi_{0}\right), v\right)+a_{4} \operatorname{cn}\left(\chi\left(\xi_{1}-\xi_{0}\right), v\right)}} \text {, }
$$

where

$$
\begin{aligned}
& a_{1}=h_{1} a_{3}+s_{1} a_{4}, a_{2}=h_{1} a_{4}-s_{1} a_{3}, a_{3}=-s_{1}-\frac{s_{2}}{v_{1}}, a_{4}=h_{1}-h_{2} \\
& E=\frac{\left(h_{1}-h_{2}\right)^{2}+s_{1}{ }^{2}+s_{2}{ }^{2}}{2 s_{1} s_{2}}, \\
& v_{1}=E+\sqrt{E^{2}+1}, \chi=\frac{s_{2} \sqrt{\left(a_{3}^{2}+a_{4}^{2}\right)\left(v_{1}^{2} a_{3}^{2}+a_{4}^{2}\right)}}{a_{3}^{2}+a_{4}^{2}}, v^{2}=\frac{v_{1}{ }^{2}-1}{v_{1}^{2}} .
\end{aligned}
$$

Solution V: When $D_{2}<0, D_{3}=0, D_{4}=0, F(v)=\left[(v-l)^{2}+h^{2}\right]^{2}$, we obtain

$$
u_{8}=\frac{C_{0}}{\omega+h \tan \left[h\left(\xi_{1}-\xi_{0}\right)\right]+l}
$$

Solutions VI When $D_{2}>0, D_{3}=0, D_{4}=0, E_{2}>0, F(v)=(v-\mu)^{2}(v-\rho)^{2}$,

$$
\begin{aligned}
& u_{9}=\frac{C_{0}}{\omega+\frac{\rho-\mu}{2}\left[\tanh \frac{(\mu-\rho)\left(\xi_{1}-\xi_{0}\right)}{2}-1\right]+\rho}, \\
& u_{10}=\frac{C_{0}}{\omega+\frac{\rho-\mu}{2}\left[\operatorname{coth} \frac{(\mu-\rho)\left(\xi_{1}-\xi_{0}\right)}{2}-1\right]+\rho} .
\end{aligned}
$$


where

Solution VII: When $D_{4}=0, D_{2} D_{3}<0, F(v)=(v-\beta)^{2}\left[(v-h)^{2}+s^{2}\right]$

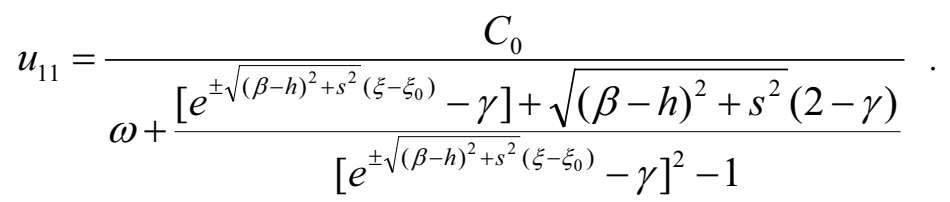

$$
\gamma=\frac{\beta-2 h}{\sqrt{(\beta-h)^{2}+s^{2}}}, \quad \delta=\sqrt{(\beta-h)^{2}+s^{2}}-\frac{\beta(\beta-2 h)}{\sqrt{(\beta-h)^{2}+s^{2}}}
$$

Solution VIII:When $D_{4}<0, D_{2} D_{3} \geq 0, F(v)=(v-\mu)(v-\rho)\left[(v-h)^{2}+s^{2}\right]$,

where

$$
u_{12}=\frac{C_{0}}{\omega+\frac{f_{1} c n\left(\frac{\sqrt{-2 s v_{1}(\mu-\rho)}}{2 v v_{1}}\left(\xi_{1}-\xi_{0}\right), v\right)+f_{2}}{f_{3} c n\left(\frac{\sqrt{-2 s v_{1}(\mu-\rho)}}{2 v v_{1}}\left(\xi_{1}-\xi_{0}\right), v\right)+f_{4}}}
$$

$$
\begin{aligned}
& f_{1}=\frac{1}{2}(\mu-\rho) f_{3}-\frac{1}{2}(\mu-\rho) f_{4}, f_{2}=\frac{1}{2}(\mu+\rho) f_{4}-\frac{1}{2}(\mu-\rho) f_{3} \quad, f_{3}=\mu-h-\frac{s}{v_{1}}, \\
& f_{4}=\mu-h-s v_{1}, G=\frac{s^{2}+(\mu-h)(\rho-h)}{s(\mu-\rho)}, v_{1}=G \pm \sqrt{G^{2}+1} \cdot v^{2}=\frac{1}{1+v_{1}^{2}} .
\end{aligned}
$$

\section{Expression of solutions}

In this section, we give concert parameters to obtain the some expression of the solutions. And we draw the graphs of some typical solutions.

Situation 1:

We take $\mu=1, \rho=-3, C_{1}=0, \omega=1, C_{0}=-\frac{9}{2}, d_{0}=\frac{48}{379}$ to get the solution

$$
u_{1}(x, t)=\frac{5-10\left(\frac{-3 \sqrt{3}}{2}-1\right)^{2}(x+t)^{2}}{-2+8\left(\frac{-3 \sqrt{3}}{2}-1\right)^{2}(x+t)^{2}} .
$$

We take $h=1, l=0, C_{1}=0, \omega=1, C_{0}=-\frac{3}{2}, d_{0}=\frac{123}{108}$ to get the solution

$$
u_{2}(x, t)=\frac{3}{-2+2 \tan \left[\left(-\frac{\sqrt{3}}{2}-1\right)(x+t)\right]} .
$$

The graphs of the solutions $\mathrm{u} 1$ and $\mathrm{u} 2$ can be seen in Figure 1 and 2. 


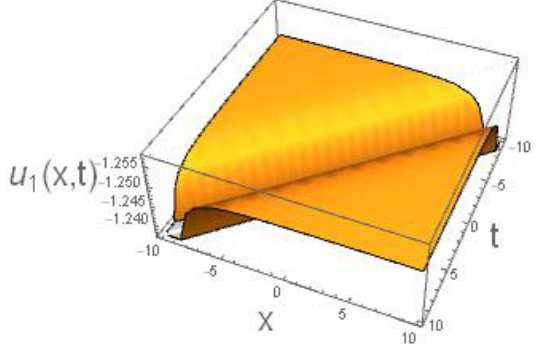

Fig. 1. Solution of . $u_{1}(x, t)$

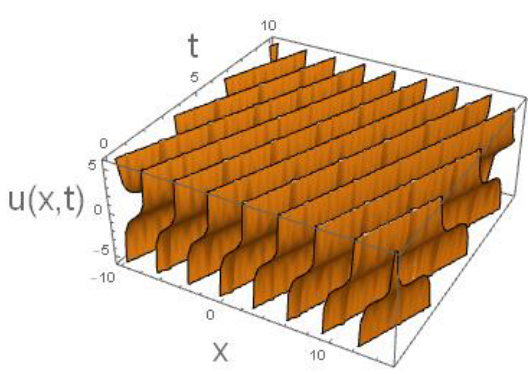

Fig. 2. Solution of $u_{2}(x, t)$.

Situation 2:

We take $\mu=1, \rho=-1, C_{1}=0, \omega=1, C_{0}=\frac{3}{2}, d_{0}=\frac{174}{11} \cdot t_{0}=\frac{3^{\frac{1}{8}}}{2^{\frac{4}{3}}}, C_{1}=0$ to get the solutions

$$
\begin{aligned}
& u_{3}(x, t)=\frac{3}{2\left[\operatorname{coth}\left[\left(\frac{\sqrt{3}}{2}-1\right)(x+t)-1\right] .\right.} \\
& u_{4}(x, t)=\frac{3}{2\left[\tanh \left[\left(\frac{\sqrt{3}}{2}-1\right)(x+t)-1\right]\right.}
\end{aligned}
$$

The graphs of the solutions $\mathrm{u} 3$ and $\mathrm{u} 4$ can be seen in Figure 3 and 4.

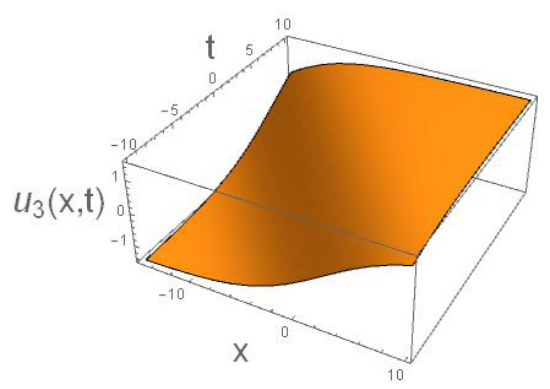

Fig. 3. solution of . $u_{4}(x, t)$.

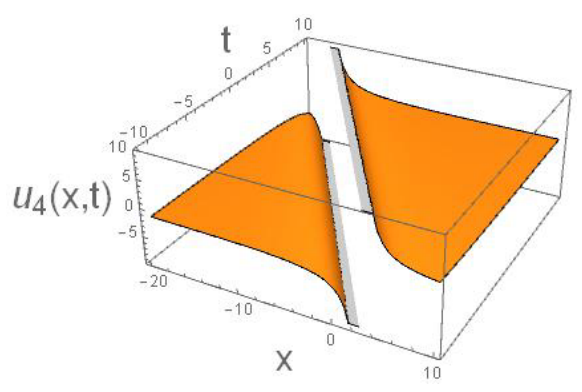

Fig. 4. solution of $u_{5}(x, t)$.

\section{Situation 3 :}

We take $\beta_{1}=-1, \beta_{2}=-1, \beta_{3}=1, \beta_{4}=1, \omega=1, C_{1}=0, C_{0}=-\frac{1}{2}, d_{0}=-\frac{24}{73}$ to get the solution

$$
u_{5}(x, t)=-\frac{s n^{2}\left[\left(-\frac{\sqrt{3}}{6}\right)(x+t), 1\right]+1}{4}
$$


Taking $C_{1}=0, d_{0}=\frac{96}{19}, a_{1}=4-\sqrt{10}, a_{4}=2, \chi=\frac{\sqrt{220+24 \sqrt{10}}}{18-4 \sqrt{10}}, a_{2}=\sqrt{10}, a_{3}=2-\sqrt{10}$, $h_{1}=1, s_{1}=1, s_{2}=1, v_{1}=3+\sqrt{10}, v=\frac{18+6 \sqrt{10}}{19+6 \sqrt{10}} h_{2}=-1, C_{0}=-\frac{1}{2}, \omega=1$.

The equation can be wrote

$$
u_{6}(x, t)=\frac{1}{2-\frac{(8+2 \sqrt{10}) \operatorname{sn}\left(\chi\left(-\frac{\sqrt{3}}{6}-1\right)(x+t), v\right)+2 \sqrt{10} \operatorname{cn}\left(\chi\left(-\frac{\sqrt{3}}{6}-1\right)(x+t), v\right)}{(2-\sqrt{10}) \operatorname{sn}\left(\chi\left(-\frac{\sqrt{3}}{6}-1\right)(x+t), v\right)+2 \operatorname{cn}\left(\chi\left(-\frac{\sqrt{3}}{6}-1\right)(x+t), v\right)}}
$$

The graphs of the solutions $\mathrm{u} 5$ and $\mathrm{u} 6$ can be seen in Figure 5 and 6.

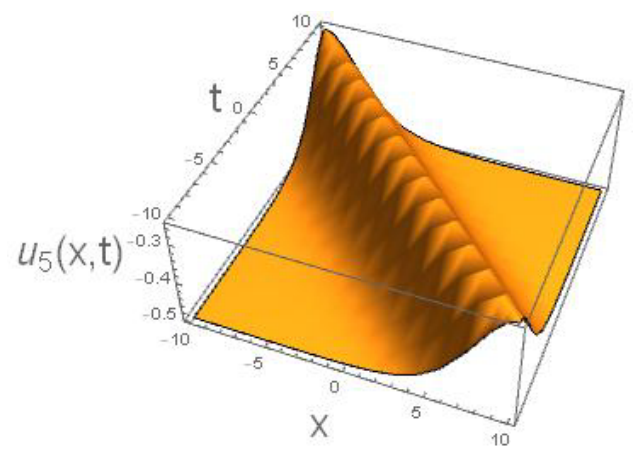

Fig. 5. solution of $u_{5}(x, t)$.

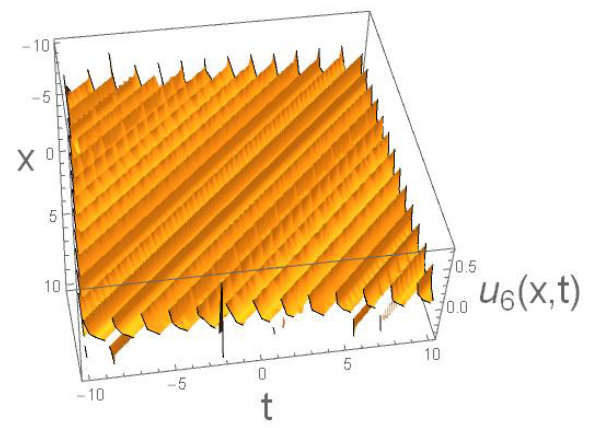

Fig. 6. solution of $u_{6}(x, t)$.

\section{Conclusion}

In this paper, we gained a series of solutions of the Green-Naghdi equa tions using the trial equation method. Besides solitary waves pattern, blow-up patterns, periodic wave patterns and periodic blow-up patterns and double pe riodic waves patterns there are two new patterns which are singular patterns and double periodic patterns. We realize them in figures with concrete param eters which help us to understand the dynamics behaviors of the Green-Naghdi model.

\section{References}

1. Z. S. Wen, Bifurcations and Exact Traveling Wave Solutions of the Celebrated GreeniNaghdi Equations. International journal of bifurcation and chaos. 7 (2017) 27.

2. F. Serre, Contribution Ul'Wtude des Wcoulements permanents et variables dans les canaux La Houille Balance. 8 (1953) 374-388.

3. C. H. Su, C. S. Gardner, Korteweg-de Vries equation and generalizations. J. Math. Phys. 10 (1969) 536-539.

4. A. E. Green, P. M. Naghdi. A derivation of equations for wave propagation in water of variable depth. J. Fluid. Mech. 78 (1976) 237-246. 
5. F. J. Seabra-Santos, D. P. Renouard, A. M. Temperville, Numerical and experimental study of transformation of a solitary wave over a shelf or isolated obstacle. J. Fluid Mech. 176 (1987) 117-134.

6. F. Dias, P. Milewski. On the fully-nonlinear shallow-water generalized Serre equations. Phys. Lett. A. 374 (2010) 1049-1053

7. O. Le MWtayer, S. Gavrilyuk, S. Hank, A numerical scheme for the Green Naghdi model. J. Comp. Phys. 229 (2009) 2034-2045.

8. C. S. Liu, Exact Traveling Wave Solutions for a Kind of Generalized Ginzburg-Landau Equation. Commun. Theor. Phys. 43 (2005) 787-790.

9. C. S. Liu, X. H. Du, Coupling Klein-Gordon-Schr dinger equation new exact solution. Acta. Phys. 54 (2005) 1039-1043.

10. C. S. Liu, Exact travelling wave solution for $(1+1)$-dimentional dispersive long wave equation. Chin. Phys. Soc. 14 (2005) 1710-1716.

11. C. S. Liu, Equivalent construction of the infinitesimal time translation op erator in algebraic dynamics algorithm for partial differential evolution e quation. Science China Physics 53 (2010) 1475-1480.

12. C. S. Liu, Exactly solving some typical RiemanniLiouville fractional mod els by a general method of separation of variables. Commun. Theor. Phys. 72 (2020) 50-55.

13. C. S. Liu, Using trial equaiton method to solve the exact soutions of variable coefficients nolinear development equation. Chin. Phys. 10 (2005) 4506- 4510.

14. C. S. Liu, Classification of all single travelling wave solutions to Calogero Focas equation. Commun. Theor. Phys. 48 (2007) 601-604

15. C. S. Liu, New exact envelope traveling wave solutions of high-order disper sive Cubic-Quintic nonlinear schrodinger equation. Commun. Theor. Phys. 44(2005) 799-801.

16. H. T. Wei, Stationary envelope solutions of a nonlinear schr"odinger-type equation. Optik. 230 (2021) 166351.

17. S. X. Liang, J. Z. Zhang, A complete discrimination system for polynomials with complex coefficients and its automatic generation. Science in China Series E. 42 (1999) 113-128.

18. L. Yang, X. R. Hou, Z. B. Zeng, A complete discrimination system for polynomials. Science in China Series E. 05 (1996) 424-441.

19. J. Z. Zhang, S. X. Liang, Complex coefficients complete discrimination system for polynomials and Auomatic forming. Science in China Series E. 01 (1999) 61-75.

20. C. S. Liu, Applications of complete discrimination system for polynomial for classifications of traveling wave solutions to nonlinear differential equations. Comput. Phys. Commun. 181 (2010) 317-324. 